Recherches en didactique des langues et des cultures

Les cahiers de l'Acedle

$15-3 \mid 2018$

Quelles médiations en didactique des langues et des cultures?

\title{
Quelle(s) langue(s) médiatrice(s) choisir en classe de langue étrangère?
}

Représentations de futurs enseignants de FLE

\section{Rea Lujić}

\section{OpenEdition}

\section{Journals}

Édition électronique

URL : http://journals.openedition.org/rdlc/3764

DOI : $10.4000 /$ rdlc.3764

ISSN : $1958-5772$

Éditeur

ACEDLE

\section{Référence électronique}

Rea Lujić, «Quelle(s) langue(s) médiatrice(s) choisir en classe de langue étrangère ? », Recherches en didactique des langues et des cultures [En ligne], 15-3 | 2018, mis en ligne le 01 septembre 2018, consulté le 19 avril 2019. URL : http://journals.openedition.org/rdlc/3764 ; DOI : 10.4000/rdlc.3764

Ce document a été généré automatiquement le 19 avril 2019

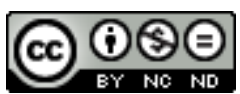

Recherches en didactique des langues et des cultures is licensed under a Creative Commons AttributionNonCommercial-NoDerivatives 4.0 International License 


\section{Quelle(s) langue(s) médiatrice(s) choisir en classe de langue étrangère ?}

Représentations de futurs enseignants de FLE

\section{Rea Lujić}

\section{Introduction}

1 Tout étudiant en langue étrangère se formant au métier de professeur de langues s'est certainement confronté au cours de ses études aux questions suivantes: Puis-je communiquer en langue maternelle au sein d'une classe de langue étrangère? Si oui, dans quels buts et dans quelle proportion? Ces questions ne sont pas nouvelles, de surcroit, on les pose dès l'aube de l'enseignement organisé de langues étrangères ou secondes (Castellotti, 2001: 12), encore aujourd'hui l'avis des spécialistes, mais aussi des nonspécialistes en didactique des langues (élèves, parents, etc.), est partagé.

2 Les partisans du paradigme linguistique dit monoglossique, dont le principe central est d'empêcher le contact des langues, considèrent l'emploi de n'importe quelle langue en classe de langue autre que la langue cible comme néfaste pour l'apprentissage de la langue cible et préfèrent les bannir des classes pour enlever la possibilité des transferts négatifs qui pourraient freiner voire nuire à l'apprentissage et provoquer une production langagière dite non-pure. Les pratiques de classe dans une approche unilingue, inspirées par les principes de ce paradigme, sont en effet des pratiques de classe issues de la méthode directe, aujourd'hui majoritairement pratiquées dans certaines écoles de langues ${ }^{1}$.

3 En ce moment, c'est plutôt le paradigme «hétéroglossique " qui se développe et un certain effort est fait pour que ses principes soient appliqués dans le contexte de l'enseignement-apprentissage des langues. Au lieu de bannir les langues non-cibles, ses partisans, en Europe (cf. Duverger, 2007 ; Candelier et Castellotti, 2013; Beacco et al., 2015), ou ailleurs (cf. Garcìa et Wei, 2014), acceptent voire encouragent l'emploi d'autres 
langues que la langue cible dans l'enseignement-apprentissage des langues parce qu'ils les trouvent bénéfiques. Un des moments clés dans le développement de cette nouvelle perspective dans le champ du bilinguisme, était l'hypothèse de Grosjean (1993) selon laquelle le bilingue n'est pas deux monolingues mais un tout qui a sa propre compétence linguistique et qui doit donc être analysé en tant que tel (p.16) ce qui concorde bien avec l'hypothèse de la multi-compétence de Cook (voir Cook, 1992 ; 2007). Dans cette optique, l'alternance codique d'une personne bilingue n'est plus étudiée comme un discours erroné, par contre, on a proposé le terme «le parler bilingue $»^{2}$ pour désigner un parler propre aux bilingues (Grosjean, 1993; Lüdi \& Py, 2003). Les modèles théoriques de l'acquisition de plusieurs langues comme le Modèle Dynamique du Multilinguisme (Herdina \& Jessner, 2002) d'une part, qui ont mis en évidence l'impact positif de l'influence crosslinguistique, et les théoriciens qui ont relevé la question de l'identité dans le contexte scolaire multilingues (Cummins \& Early, 2011) d'autre part, ont également beaucoup contribué au développement de cette perspective et influencé les changements dans les pratiques de classe.

4 Les activités du Conseil de l'Europe, entreprises durant les deux dernières décennies dans le contexte de l'enseignement des langues, montrent que la politique linguistique européenne est dans la lignée de ces derniers. Ses créateurs ont investi beaucoup d'effort dans la promotion de la politique européenne du plurilinguisme et du pluriculturalisme (voir Coste, Zarate \& Moore, 1997 ; Candelier et al., 2012 ; Beacco et al., 2015), et c'est l'approche plurilingue (voir Candelier \& Castelotti, 2013), une nouvelle approche didactique, qui met en œuvre des théories et des politiques comme celles mentionnées cidessus. Ceci se fait notamment en didactisant l'alternance en classe de langue (voir Duverger, 2007). Par exemple, un enseignant peut proposer aux élèves à la fin de son cours de produire un résumé du cours en langue maternelle, ou encore il peut leur donner des textes dans leur première langue étrangère comme étayage pour écrire en deuxième langue étrangère.

5 En Croatie, cette politique est, dans les discours, acceptée au niveau national et officiel. Par exemple, dans le programme national pour le primaire $(2005)^{3}$, il est fait mention du plurilinguisme et du pluriculturalisme, notamment en acceptant l'emploi de la langue maternelle en classe de langue étrangère pour des raisons métalinguistiques. Aussi, selon la Loi sur l'éducation et l'enseignement au primaire et au secondaire (NN 87/08) l'Enseignement d'une Matière Intégrée à une Langue Etrangère (EMILE) en Croatie est

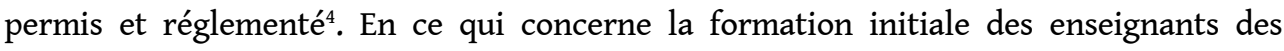
langues, il existe dans toutes les universités plusieurs cours qui traitent ce sujet, surtout au niveau du Master. Par ailleurs, le double Master ${ }^{5}$ est certainement une des spécificités de l'enseignement supérieur croate qui favorise le développement du plurilinguisme dans les écoles croates. Après avoir fini leurs études, les enseignants croates continuent leur formation tout au long de leur vie, et il y en a qui participent aux activités organisées autour du Centre européen pour les langues vivantes (CELV) ${ }^{6}$. De plus, le plurilinguisme devient un sujet de plus en plus populaire parmi les chercheurs croates (voir Medved Krajnović, 2010 ; Udier \& Cergol Kovačević, 2015).

6 Même si on connaît assez bien l'état des lieux au niveau officiel et théorique, on connaît beaucoup moins la situation dans la classe de langue. Les enseignants introduisent-ils dans leurs pratiques de classe les savoirs récents sur le bilinguisme, plurilinguisme et l'acquisition de plusieurs langues? Que font-ils afin d'aider les apprenants à développer une compétence plurilingue et pluriculturelle? Quelles sont les langues médiatrices dont 
les enseignants se servent dans l'enseignement des langues étrangères, surtout dans l'enseignement de la troisième langue ? Les études effectuant un état des lieux, comme par exemple, à propos de l'attitude des enseignants ou des apprenants croates vis-à-vis de l'alternance codique en classe de langue, ou sa représentation (fréquence, forme etc.) en classe de langue, sont rares (voir Čurković-Kalebić, 2003). Les chercheurs qui s'intéressent à la mise en œuvre de l'approche plurilingue sont aussi peu nombreux (voir Hudi \& Vrhovac, 2007 ; Lujić, 2016).

7 Sur ce point, nous sommes d'avis, comme Giroux (2016), qu'il faut absolument connaître l'attitude des enseignants de langue sur ces sujets parce que leur attitude influencera leurs pratiques de classe ce qui, par conséquent, influencera les résultats de l'enseignement/apprentissage. En dépit des recommandations officielles, politiques supranationales ou nationales, ce sont essentiellement les enseignants qui restent les maîtres du jeu : maîtres d'accepter ou non la langue première, maîtres de l'utiliser ou non eux-mêmes pour certains usages, maîtres de lui conférer un rôle plus ou moins important dans l'apprentissage et, ce faisant, de renforcer son éviction ou de la réhabiliter aux yeux des apprenants (Castellotti, 2001 : 49). Ainsi, il semble tout à fait pertinent de s'interroger sur les attitudes ou les représentations des futurs enseignants de langues étrangères face à la question des langues médiatrices en classe de langue. Mais, avant d'entrer dans le vif du sujet, un travail de clarification conceptuelle s'impose sur deux notions: celle de représentation et celle d'attitude. L'attitude est généralement définie comme une disposition à réagir de manière favorable ou non à une classe d'objet (Castellotti et Moore, 2002: 7). Par attitude des étudiants de langue vers l'emploi des langues médiatrices en classe de langue, il faut entendre dans un premier temps les connaissances qu'un étudiant possède sur l'emploi de ces dernières (quelle(s) langue(s) choisir, comment les introduire en classe etc.), ensuite les ressentis qu'un étudiant a sur l'emploi des différentes langues médiatrices et, finalement, sa disposition à agir - quelle(s) langue (s) choisirait-il ? Par ailleurs, les attitudes ne peuvent pas être directement observées mais elles génèrent les comportements plus ou moins stables (Castellotti et Moore, 2002). En revanche, la représentation est un concept qui est également entré dans le champ de la didactique des langues de la psychologie sociale et y a pris une position importante. Il s'agit d'une construction à la fois stable et dynamique, influencée par différents facteurs (sociaux, historiques, idéologiques etc.). Elle sert de cadre d'exploitation des connaissances, déclenche des inférences, et guide les comportements (Castellotti et Moore, 2002). Les représentations des étudiants sont influencées par plusieurs facteurs : en premier lieu par leur formation initiale (le contenu des cours, l'attitude des professeurs, l'expérience dans les écoles observées etc.), et par la prise de position supranationale et nationale présentée dans les documents officiels (curriculum scolaire etc.), mais dépend aussi de leur propre expérience en tant qu'apprenant et de l'opinion publique sur ce sujet ${ }^{7}$. Pour mieux comprendre leurs représentations il est surtout important de mentionner qu'au cours de leur formation initiale, le sujet étudié a été abordé à plusieurs reprises, dans les différents cursus et sous différents angles. Pour n'en mentionner que quelque uns: la place et le rôle des langues médiatrices à travers l'histoire de la didactique des langues, le statut de la langue maternelle aujourd'hui, la politique linguistique de l'Union Européenne, les approches plurielles, etc.

8 L'intention de ce papier n'est pas de donner un regard critique ni de faire un état des lieux, mais plutôt d'essayer de comprendre les représentations des étudiants en ce qui concerne le choix des langues médiatrices en classe de FLE, ce qui, par conséquent, 
pourrait influencer leurs pratiques de classe qu'ils mettraient en place un jour tant qu'enseignants. Pour ce faire nous nous sommes posé les questions suivantes: quelles sont les représentations des étudiants croates en Master FLE, c'est-à-dire de futurs enseignants du FLE, sur cette problématique. Considèrent-ils l'emploi des langues noncibles de la part de l'enseignant d'un point de vue négatif ou positif et pourquoi ?

\section{Cadre de recherche}

La plupart des travaux portant sur ce sujet sont des articles de synthèse qui font le point sur l'état des connaissances scientifiques dans ce domaine (voir Dabène, 1987 ; Castellotti, 2001; Giroux, 2016). Autrement dit, ils ne reposent pas sur des expérimentations. Parmi les articles de recherche, rares sont ceux qui ont analysé le point de vue sur ce sujet des enseignants ou des apprenants (voir Wantz-Bauer, 2001). Il y a quand même un certain nombre de travaux récents sur le rôle de l'anglais comme langue passerelle vers le français, à la fois du point de vue des pratiques et des représentations (notamment, ceux de Gilles Forlot). Et au cours des années 1990, il y a eu aussi des travaux sur la façon dont les enseignants acceptaient au non la présence de la L1 en classe de L2 (voir Castellotti, 1997).

Par conséquent, on a choisi d'analyser le point de vue des étudiants en Master FLE sur l'emploi des langues autres que la langue cible en classe da langue étrangère. La nécessité d'en faire une recherche est apparue lors de l'analyse de leurs journaux de bord qui font partie intégrante de leur cursus « Stage d'observation et de pratiques de classe ». Le choix des langues fait par l'enseignante s'est avéré comme la pratique de classe qui a attiré le plus leur attention. De chaque cours observé, ils ont mentionné à plusieurs reprises la langue choisie par l'enseignante et dans quelle pratique de classe. Il est important de préciser que cet aspect de l'analyse du cours ne leur a pas été imposé ; ils devaient décrire les cours observés, et ils étaient encouragés de donner leurs points de vue sur les pratiques de classe observées.

Les étudiants (9) ayant participé à cette recherche étaient inscrits en Master 2 FLE à l'Université de Zadar en Croatie. Ils sont spécialistes de la didactique du français langue étrangère, mais suivaient aussi les cours de Master 2 d'une autre langue étrangère (l'anglais, l'espagnol ou l'italien). Alors, ils maîtrisent le français ainsi qu'une autre langue étrangère à un niveau avancé, mais aussi, à un niveau débutant ou intermédiaire cette fois une troisième langue étrangère.

Ils ont observé des cours dans quatre écoles primaires où le français est enseigné en tant que cours facultatif que les élèves peuvent choisir en $4 \mathrm{eme}$ classe ${ }^{8}$. Étant donné qu'en Croatie les élèves apprennent une langue étrangère dès le début de leur scolarisation obligatoire ${ }^{9}$, pour eux le français est en effet une troisième langue.

Les enseignantes observées ont également suivi et validé un Master double cursus, alors elles maîtrisent au moins trois langues, souvent quatre: le croate, deux langues étrangères à un niveau avancé, le français et une autre langue (l'italien, l'anglais ou l'allemand) qu'elles enseignent aussi. De même, à un niveau débutant ou intermédiaire elles maîtrisent une quatrième langue.

14 Ainsi, on peut d'ores et déjà constater que la recherche s'est déroulée dans un contexte plurilingue. 


\section{Analyse et résultats}

Les matériaux empiriques de cette étude se composent de neuf journaux de bord de neuf étudiants de Master 2 FLE qui ont été produits aux cours du «Stage d'observation et de pratiques de classe ». Les journaux sont d'une longueur approximative de 20 pages standard. Pour en faire l'analyse qualitative, nous avons dégagé de notre corpus toutes les formulations des étudiants relatives à l'emploi des langues choisies. Nous en avons relevé 80. Après les avoir toutes lues, nous avons procédé à une double classification des commentaires, tout d'abord selon la langue choisie par l'enseignant et ensuite selon la fonction que la langue mentionnée occupe dans une situation de classe. Selon le premier critère, la langue choisie, quatre catégories ont émergé : l'emploi de la langue maternelle (le croate), l'emploi de la première langue étrangère (l'anglais), l'emploi de la langue cible (le français) et l'alternance codique. Après avoir classifié les commentaires selon le deuxième critère, on a pu identifier les quatre catégories suivantes : la langue en tant qu'outil métalinguistique (ex. Difficile, c'est le contraire de facile), la langue en fonction de l'outil qui assure le déroulement de la séance (ex. Ouvrez vos cahiers!), le discours ritualisé (ex. Bravo !) et la langue pour introduire le contenu du cours (ex. Quels sont les personnages principaux du texte?). Pour mieux comprendre les représentations des étudiants, nous avons essayé d'interpréter leurs observations à partir du contexte donné. Dans l'exemple suivant, un étudiant défend le choix de l'enseignant avec une argumentation solide et de cette manière il exprime son point de vue :

E : Pour que les élèves puissent comprendre son explication donnée en français, l'enseignante ensuite donne une explication en croate.

\section{La langue maternelle en classe de la deuxième langue étrangère}

L'analyse des journaux de bord nous a permis de dégager deux fonctions principales de la langue maternelle, ici le croate, dans la classe de FLE : celle du relais métalinguistique et celle du facilitateur de la compréhension des instructions de travail. Les commentaires révèlent que la langue maternelle servait aux enseignantes observées, du point de vue des étudiants, pour donner aux élèves des explications métalinguistiques, plus précisément, pour mieux expliquer le nouveau contenu lexical ou grammatical, ce qui est, selon Castellotti (2001), une des rares possibilités d'emploi de la langue première légitimée par l'institution (p. 19).

Un étudiant a noté :

E : Dans la partie introductive du cours, l'enseignante s'est servie du croate pour expliquer la formation des questions en français.

Ensuite, il justifie ce recours au croate :

$\mathrm{E}$ : Elle [enseignante] a expliqué ces règles en croate parce que les élèves sont débutants.

Ces explication et prise de position concordent bien avec l'avis des spécialistes en la matière (voir Castellotti, 2001) : la langue maternelle est plus acceptée dans les niveaux débutants que dans les niveaux avancés.

Recherches en didactique des langues et des cultures, 15-3 | 2018 
Les commentaires révèlent aussi que, selon les étudiants, les enseignantes recouraient au croate quand il leur était clair que les explications données en français ne suffisaient pas aux élèves, ou seulement pour s'assurer de leur compréhension :

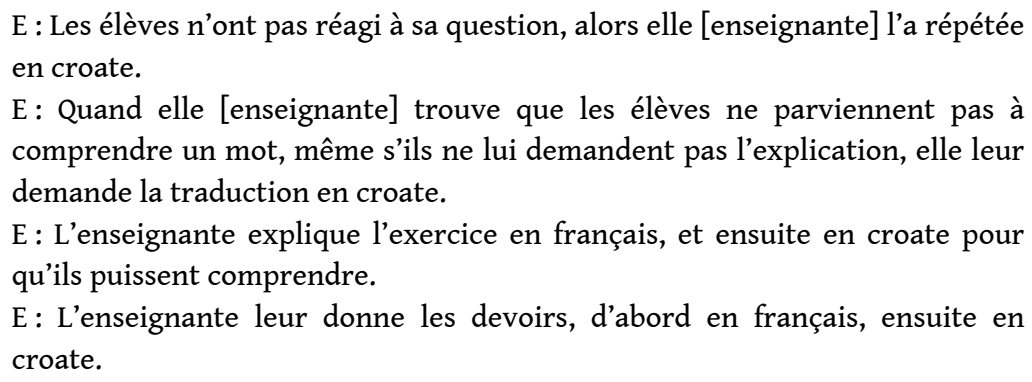

19 Dans ces notes on ne trouve pas de trace de critique, mais plutôt une description neutre. Même si les enseignantes observées n'ont pas banni la langue maternelle, les notes montrent qu'il y en a qui y recouraient comme dernier moyen d'explication, après avoir épuisé d'autres moyens linguistiques et extralinguistiques, tels que la paraphrase, le synonyme ou les gestes :

E : Si les élèves ne comprennent pas son explication en français, ni avec l'aide de la paraphrase ni celle des gestes, elle [enseignante] leur explique en croate.

20 Ces exemples montrent que ce sentiment de culpabilité décrit chez Dabène (1987) qui se manifestait à l'époque lors de l'emploi de la langue maternelle en classe de langue étrangère n'est pas encore disparu, et d'autre part, que ce sentiment ne semble pas s'accorder avec l'attitude de futurs enseignants de langue en Croatie. Bien au contraire, un étudiant a bien argumenté l'importance de la langue maternelle :

E : Parfois, il n'y a aucun sens à parler en français si les enfants ne vous comprennent pas. Dans cette situation il semble plus important de transmettre le message et que les enfants soient détendus. Si vous insistez sur le français, les enfants peuvent devenir anxieux.

\section{La première langue étrangère en classe de deuxième langue étrangère}

21 Comme on l'avait indiqué dans la définition de notre cadre de recherche, tous les participants à cette recherche maîtrisaient l'anglais, souvent à un niveau plus avancé que le français. Ainsi, cette langue, dans l'optique hétéroglossique, pouvait leur servir d'outil précieux. Pourtant, les notes révèlent que seulement une enseignante a, quelque fois, choisi l'anglais, comme langue médiatrice :

E : Un élève lui [enseignante] a demandé comment dire les parents en français, et elle lui a répondu comment cela se dit en anglais.

$\mathrm{E}$ : Les élèves demandent comment dire l'éléphant. Elle [enseignante] leur répond de le dire en anglais mais avec une prononciation française.

22 Même si c'était rare, au moins selon les notes des étudiants, ces deux exemples montrent les possibles bénéfices de la première langue étrangère: elle pourrait leur servir d'étayage ou de stratégie d'apprentissage dans le contexte de l'enseignement/ 
apprentissage d'une deuxième langue étrangère. Ce bénéfice a été plusieurs fois rapporté dans le journal de bord de l'étudiant qui a observé les cours de cette enseignante, ce qui probablement, un jour, influencera ses pratiques de classe :

$\mathrm{E}$ : C'est très utile d'employer l'anglais en classe de français.

D'un autre côté, on ne sait pas si les autres étudiants ont eu l'occasion d'observer cette pratique, mais l'absence de commentaires suggère que la première langue étrangère n'y était pas beaucoup ou pas du tout représentée, ce qui pourrait influencer leurs pratiques de classes futures.

\section{La langue cible en tant que langue médiatrice}

Les notes des étudiants sur l'emploi de la langue cible, dans notre cas du français, se révèlent importantes parce qu'elles peuvent démontrer les attentes des étudiants et ainsi démasquer leurs représentations de l'emploi (rôle et fréquence) de la langue cible. L'analyse des commentaires suggère que les enseignantes se servaient du français en tant que langue médiatrice pour: inciter les élèves à la communication, pour gérer la communication et pour gérer les activités et la classe. Leurs commentaires sur l'emploi de la langue cible dans le discours fortement ritualisé ou pour gérer la classe ont été assez nombreux :

$\mathrm{E}$ : L'enseignante utilise très souvent le français, elle s'en sert à n'importe quel moment, par exemple Qui est absent, Alors on y va, etc.

E : Les phrases comme C'est fini !, Bonjour tout le monde !, Merci !, Bravo!, sont toujours en français.

Un étudiant argumente son opinion positive vers cette pratique de classe :

$\mathrm{E}:$ Ce type de discours les enfants ont dû bien le mémoriser et le comprendre parce qu'on l'entend tous les jours.

Les questions, le moyen le plus important pour inciter les élèves à la communication, formulées en français, ont été fortement représentées dans leurs commentaires :

$\mathrm{E}$ : Elle [enseignante] introduit la leçon en leur posant les questions en français : Qui participe à la promenade ? Quel temps fait-il ?

$\mathrm{E}$ : Elle [enseignante] leur pose des questions uniquement en français : Où est Ivan? Où est Alan? Qu'est-ce qu'ils font?

La surprise du deuxième étudiant sur ce choix de langue utilisée pour cet objectif a été bien marquée par le choix de l'adverbe uniquement. Une grande importance donnée à la langue cible de la part des enseignantes, ainsi que la surprise des étudiants de cette pratique, ont été aussi dévoilées par la fréquence de commentaires de type suivant :

$\mathrm{E}$ : Elle [enseignante] ne leur donne pas les réponses en croate mais elle utilise les synonymes et explique uniquement en français.

E: Ils ont répondu en croate, mais l'enseignante a insisté pour qu'ils répondent en français.

$\mathrm{E}:$ Elle [enseignante] traduit son énoncé et ensuite demande de le répéter.

$\mathrm{E}$ : Après avoir demandé quelque chose en croate, elle [enseignante] lui répond en français.

A partir des leurs commentaires nous pouvons émettre deux hypothèses. D'un côté, il est possible que les étudiants admirent l'enseignant car employer la langue cible dans toutes 
ces situations exige certainement plus d'effort de sa part. De plus, il est possible qu'ils ne se considèrent pas encore assez formées pour pouvoir travailler de cette manière. D'un autre côté, il est possible qu'ils ne s'y attendaient pas à cause de leurs expériences personnelles en tant qu'élèves. Enfin, il semble qu'ils considèrent ces pratiques très utiles :

E : Pour les élèves, il est vraiment utile que l'enseignante utilise de cette manière le français.

Un autre étudiant a bien élaboré son attitude positive en prenant en considération le statut du français en Croatie :

E : Etant donné que les élèves n'ont le cours de français que deux fois par semaine, en Croatie c'est la seule occasion d'entendre cette langue.

Une autre a expliqué son attitude en utilisant un argument psycholinguistique :

E : Il faut utiliser le français le plus possible parce qu'à ce stade-là du développement cognitif ils acquièrent ce qu'ils entendent sans beaucoup d'effort.

\section{L'alternance codique en classe de langue étrangère}

Puisque les étudiants ont remarqué la présence de deux, voire trois langues dans la classe, cela implique la présence de l'alternance codique en classe de langue étrangère. Pourtant, leurs commentaires révèlent qu'elle y a trouvé place seulement, ou presque seulement, sous une seule forme - micro-alternance ce qui désigne

que durant le cours dispensé et structuré majoritairement en l'une des deux

langues, on aura recours, ponctuellement et de manière non programmée, à l'usage de l'autre langue (Duverger, $2007: 84$ ).

Un étudiant a noté :

E : Les explications ont été en croate avec quelques mots français.

Evidemment, l'alternance lui a servi d'explication métalinguistique, ce qui est, selon Gajo (2000) une des fonctions principales de l'alternance initiée par l'enseignant. Un tel commentaire, neutre, ne révèle pas beaucoup sur sa prise de position. Pourtant, déjà le fait d'avoir été témoin de ce type de l'alternance, augmente la probabilité qu'elle s'en serve un jour en tant qu'enseignante.

Bien que la micro-alternance ait été présente pendant les cours, les étudiants n'ont pas noté, la présence de la méso-alternance qui est

opérée par le professeur pendant le cours de manière raisonnée, réfléchie, volontaire, sous forme de séquences successives et ceci, dans la perspective de favoriser chez les élèves la mise en œuvre des processus d'apprentissage (Duverger, $2007: 84)$.

Pourtant, c'est l'introduction de la méso-alternance en classe de langue qui rend claire la distinction entre une classe monolingue et une classe plurilingue. 


\section{Conclusion}

L'objectif de cette étude était de connaître les représentations de futurs enseignants de français langue étrangère des langues médiatrices en classe de langue étrangère dans un contexte d'enseignement-apprentissage du FLE. Après l'analyse de leurs commentaires présentés dans leurs journaux de bord, il a été possible de dégager les conclusions suivantes :

- les étudiants trouvent que la classe devrait se faire en langue cible autant qu'il est possible de le faire ; c'est encore plus important dans des pays où les langues enseignées-apprises ne font pas partie du paysage linguistique

- dans le cas où les élèves ne comprennent pas le discours de l'enseignant en langue cible, il est nécessaire de recourir à la langue source, surtout quand on enseigne aux élèves débutants, mais après avoir épuisé d'autres moyens (linguistiques et extralinguistiques)

- les autres langues médiatrices sont permises, à condition qu'elles facilitent la compréhension et encouragent l'apprentissage.

Evidemment, le «mythe du tout L2» ou «le tabou de la L1» n'est pas en train de disparaitre mais plutôt d'évoluer dans une forme plus souple. Pourtant, il semble qu'en ce moment on est loin de la didactisation de l'alternance dont l'objectif serait d'apprécier les répertoires plurilingues des élèves et de développer chez eux une compétence plurilingue et pluriculturelle. En effet, les notes d'étudiants montrent qu'ils n'ont pas vu sur le terrain comment introduire la méso-alternance en classe de langue. Par contre, ils ont vu qu'une approche monolingue est tout à fait acceptable et légitime. Ils ont pu se demander pourquoi pas alors travailler un jour autrement, en introduisant dans leurs classes une approche plurilingue. Il semble que, en dépit des efforts faits dans les politiques éducatives, enseigner/apprendre une langue étrangère continuera de signifier construire un monolinguisme nouveau tant que les enseignants ne changeront pas leurs pratiques. Et si on veut que le plurilinguisme ne soit pas qu'une tarte à la crème, le travail le plus important doit se faire à partir de la formation des enseignants de langues. Avant tout il est nécessaire de bien former les futurs enseignants, car une fois une position prise, il est ensuite difficile de changer. Il est alors important de chercher comment former les enseignants déjà en fonction à l'approche plurilingue, pour qu'ainsi ils aient le choix de la pratiquer ou non.

\section{BIBLIOGRAPHIE}

Beacco, J-C., Byram, M., Cavalli, M., Coste, D., Egli Cuenat, M., Goullier, F. \& Panthier, J. (2015). Guide pour le développement et la mise en œuvre de curriculums pour une éducation plurilingue et interculturelle. Bruxelles : Conseil de l'Europe.

Candelier, M. \& Castellotti, V. (2013). «Didactique(s) du/des plurilinguisme(s) ». In Simonin, Jacky \& Wharton, Sylvie (dir.). Sociolinguistique du contact. Dictionnaire des termes et concepts. Lyon : ENS Editions.

Recherches en didactique des langues et des cultures, 15-3 | 2018 
Candelier, M., Camilleri Grima, A., Castellotti, V., de Pietro, J-F., Lörincz I., Meissner, F-J, Schröder-Sura, A. \& Noguerol, A. (2012). Le CARAP - Compétences et ressources. Strasbourg : Conseil de l'Europe.

Castellotti, V. (1997). « Langue étrangère et langue première en milieu scolaire : didactiser l'alternance? ?. Études de linguistique appliquée, nº 108. pp. 401-410.

Castellotti, V. (2001). La langue maternelle en classe de langue étrangère. Paris : CLE International.

Castellotti, V. \& Moore, D. (2002). Représentations sociales des langues et enseignements. Guide pour l'élaboration des politiques linguistiques éducatives en Europe - De la diversité linguistique à l'éducation plurilingue. Etude de référence. Strasbourg : Conseil de l'Europe.

Cook, V. J. (1992). “Evidence for multi-competence”. Language Learning, vol. 4, n 42. pp. 557-591.

Cook, V. J. (2007). “Multi-competence: black-hole or worm-hole for second language acquisition research”. In Han, Z. (dir.). Understanding second language process. Clevedon: Multilingual Matters.

Coste, D., Moore, D. \& Zarate, G. (1997). Compétence plurilingue et pluriculturelle. Strasbourg : Conseil de l'Europe.

Cummins, J. \& Early, M. (dir.) (2011). Identity Texts: The Collaborative Creation of Power in Multilingual Schools. London: Tretntham Books.

Ćurković-Kalebić, S. (2003). Jezik i društvena situacija : Istraživanje govora u nastavi stranog jezika. Zagreb : Školska knjiga.

Dabène, L. (1987). « Langue maternelle, langue étrangère ». Quelques réflexions. Langues modernes. pp. 91-95.

Duverger, J. (2007). « Didactiser l'alternance des langues en cours de DNL ». Tréma, n 28. pp. 81-88. http://trema.revues.org/302.

Gajo, L. (2000). Immersion, bilinguisme et interaction en classe. Paris : CREDIF-Didier, Coll. LAL.

Garcìa, O. \& Wei, L. (2014). Translanguaging: Language, Bilingualism and Education. New York : Palgrave Macmillan.

Giroux, L. (2016). « La place et le(s) rôle(s) de la langue maternelle des apprenants en cours de langue étrangère ». Synergies France, no 10. pp. 55-68.

Grosjean, F. (1993). « Le bilinguisme et le biculturalisme : essai de définition ». Travaux Neuchâtelois de Linguistique (TRANEL), no 19. pp. 13-42.

Herdina, P. \& Jessner, U. (2002). A Dynamic Model of Multilingualism: Perspectives of Change in Psycholinguistic. Clevedon: Multilingual Matters.

Hudi, A. I., \& Vrhovac, Y. (2007). « Dvojezična nastava - što o tome misle učenici ». Strani jezici: časopis za unapređenje nastave stranih jezika, nํ36. pp. 63-73.

Lüdi, G. \& Py, B. (2003). Etre bilingue (3e éd.). Berne. Francfort sur Main et New York : Peter Lang. Medved Krajnović, M. (2010). Od jednojezičnosti do višejezičnosti: Uvod u istraživanje procesa ovladavanja inim jezikom. Zagreb: Leykam International d.o.o.

Udier, S. \& Cergol Kovačević, K. (dir.) (2015). Applied Linguistics Research and Methodology: Proceedings from the 2015 CALS conference. Frankfurt am Main: Peter Lang.

Vican, D. \& Milanović Litre, I. (dir.) (2005). Nastavni plan i program za osnovnu školu. Zagreb :

Ministarstvo znanosti, obrazovanja i športa. 
Wantz-Bauer, F. (2001). «Le statut de la langue maternelle en cours de langue étrangère : points de vue d'apprenants ». In Castellotti, V. (dir.). D'une langue a d'autre: Pratiques et représentations. Rouen : Publications de l'Université de Rouen.

\section{NOTES}

1. La méthode Berlitz est, en fait, une méthode directe.

2. Dans le monde anglophone on trouve son synonyme - la transcommunication (angl. translanguaging) (voir, Garcìa \& Wei, 2014).

3. Le programme national pour les écoles secondaires date du 1993 et il n'y a pas mention de plurilinguisme.

4. Pourtant, ce programme n'est réservé que pour des enfants des groupes sociaux privilégiés : les écoles offrant ce programme ne se trouvent que dans de grandes villes, ce programme n'est offert que dans des lycées privées ou publiques qui s'adressent aux meilleurs élèves issus du collège, jamais dans les lycées professionnelles ou écoles secondaires, et, finalement, l'inscription est payante.

5. Les étudiants des facultés qui forment les futurs enseignants (de langues étrangères) se spécialisent très souvent en deux matières, par exemple deux langues étrangères ce qui leur permet d'introduire pleinement les idées du Conseil de l'Europe.

6. Par exemple, le point national du CELV a organisé en 2016. une conférence portant sur CARAP; pour en savoir plus: http://www.ecml.at/Portals/1/ HtmlTagFiles/2a8e4026-6443-4656-b7ec-990480da0aed.pdf.

7. L'opinion publique sur ce sujet est très souvent formé à travers les publicités des écoles de langues et, en Croatie, la majorité des publicités mettent en avant comme une qualité incontestable le fait qu'ils offrent des cours avec des locuteurs natif ce qui forcera à parler en langue étrangère.

8. Cours Moyen deuxième année.

9. Cours Élémentaire première année.

\section{RÉSUMÉS}

La présente recherche a pour objectif d'étudier les représentations de futurs enseignants de français langue étrangère à propos des langues médiatrices en classe de langue étrangère dans un contexte d'enseignement-apprentissage du FLE en Croatie. Dans la première partie de l'article nous introduisons deux paradigmes théoriques qui traitent différemment la place des langues non-cibles en classe de langue, le paradigme monoglossique, dont le principe didactique est d'empêcher le contact des langues, et le paradigme hétéroglossique, dont les représentants trouvent le contact des langues bénéfique dans l'enseignement-apprentissage des langues. Dans la deuxième partie de l'article, à partir des analyses des journaux de bord des étudiants de Master FLE, nous essayons de faire une esquisse de leurs représentations des choix de langues 
médiatrices utilisées en classe du FLE par les enseignantes observées, et, ainsi, essayer de mieux connaître la place que le plurilinguisme pourrait prendre dans l'avenir du FLE en Croatie. L'analyse qualitative des journaux de bord a montré que, selon les étudiants, le cours du FLE devrait se faire entièrement en langue cible, et que le recours à la langue maternelle devrait être accepté seulement si les élèves ne réussissent pas à comprendre le discours de l'enseignant en langue cible. Ces résultats indiquent que le paradigme hétéroglossique et ses valeurs plurilingues dans le domaine du FLE en Croatie semblent loin d'être mis en œuvre.

This study examines the representation of future teachers of French as a foreign language (FFL) on languages which should be used to teach and to learn in the context of the FFL. In the introduction, we present two theoretical paradigms that have varying focus on the place of the non-target languages in the language classroom. The monoglossic paradigm, according to which the language contact in the classroom should be prevented as it could hinder progress in the target language and the heteroglossic paradigm, which encourages the language contact in teaching-learning languages because it can be beneficial.

In the second part of the article, we have analysed the FFL Masters students' logbooks, written throughout their practical placement in primary schools. The analysis was carried out in order to understand their representations related to the choice of the languages used in the FFL class by the observed teachers that could help us to anticipate the place that the plurilingualism could take in the future of the FFL in Croatia.

The qualitative analysis showed that, according to particular students the study was conducted on, the target language, in this case French, should be used as much as possible, and that students' mother tongue should be used only if the students fail to understand teachers' discourse in the target language. These results may indicate that the heteroglossic paradigm and its plurilingual values in the field of the Croatian FFL are far from being implemented.

\section{INDEX}

Keywords : mother tongue, mediating language, FFL, plurilingualism

Mots-clés : langue maternelle, langue médiatrice, FLE, plurilinguisme

Thèmes: Jeunes chercheur.e.s

\section{AUTEUR}

\section{REA LUJIĆ}

2017 : Ph.D. en Didactique des langues, Université de Zagreb, Croatie

2008 : M.A. en langue et littérature française / M.A. en pédagogie, Université de Zagreb, Croatie Depuis 2014 : assistante, Université de Zadar en Croatie, Département des Études Françaises et Ibero-romanes (Master FLE)

2008-2014 : professeur de FLE, École Internationale (IB), Zagreb, Croatie

Intérêts de recherche : Acquisition de plusieurs langues, Didactique du FLE, Interaction et discours dans la classe de langue, Multilinguisme/Plurilinguisme

rlujic[at]unizd.hr

Département des Études Françaises et Ibéro-romanes, section langue française et littérature 